\title{
Proteomic analysis of cerebrospinal fluid in pediatric acute lymphoblastic leukemia patients: a pilot study
}

\author{
Linghong Guo 1,2,* \\ Honghong Ren ${ }^{2}$ \\ Hao Zeng ${ }^{2}$ \\ Yanqiu Gong 3 \\ Xuelei Ma',*
}

'Department of Biotherapy, State Key Laboratory of Biotherapy and Cancer Center, West China Hospital, Sichuan University and Collaborative Innovation Center of Biotherapy, Chengdu 6I004I, People's Republic of China; ${ }^{2}$ West China School of Medicine, Sichuan University, Chengdu, People's Republic of China; ${ }^{3}$ State Key Laboratory of Biotherapy and Cancer Center, West China Hospital, Sichuan University, Chengdu, People's Republic of China

*These authors contributed equally to this work
Correspondence: Xuelei Ma Department of Biotherapy, State Key Laboratory of Biotherapy and Cancer Center, West China Hospital, Sichuan University and Collaborative Innovation Center of Biotherapy, No 37, Guoxue Alley, Chengdu 61004I, People's Republic of China

Tel/fax +86 2885475576

Email drmaxuelei@gmail.com
This article was published in the following Dove Medical Press journal: OncoTargets and Therapy

Purpose: Involvement of central nervous system in acute lymphoblastic leukemia (CNSL) remains one of the major causes of pediatric acute lymphoblastic leukemia (ALL) treatment failure. However, the current understanding of the pathological process of CNSL is still limited. This study aimed to better understand the protein expression in cerebrospinal fluid (CSF) of ALL and discover valuable prognostic biomarkers.

Materials and methods: CSF samples were obtained from ALL patients and healthy controls. Comparative proteomic profiling using label-free liquid chromatography-tandem mass spectrometry was performed to detect differentially expressed proteins.

Results: In the present study, 51 differentially expressed proteins were found. Among them, two core clusters including ten proteins (TIMP1, LGALS3BP, A2M, FN1, AHSG, HRG, ITIH4, CF I, C2, and C4a) might be crucial for tumorigenesis and progression of ALL and can be potentially valuable indicators of CNSL.

Conclusion: These differentially expressed proteins of ALL children with central nervous system involvement and normal children may work as diagnostic and prognostic factors of ALL patients.

Keywords: ALL, central nervous system leukemia, CSF, mass spectrometry, proteomics

\section{Introduction}

Acute lymphoblastic leukemia (ALL) is a malignancy derived from the medullary or lymphocyte precursor cells. ${ }^{1}$ ALL is the most common cancer in children, accounting for $25 \%-35 \%$ of pediatric cancers. ${ }^{2,3}$ With the understanding of the pathogenesis of ALL and individualized stratified therapy, the clinical efficacy of children with ALL has been significantly improved. ${ }^{4-8}$ However, central nervous system (CNS) involvement remains one of the major causes of ALL treatment failure. ${ }^{9-11}$ About $5 \%$ of newly diagnosed ALL children are found to be with CNS infiltration. ${ }^{9,12}$ Without specific treatment for these children, the prevalence rate of central nervous system leukemia (CNSL) will increase to $50 \%-70 \%$ during the whole disease process. ${ }^{13-15}$ Currently, the mechanism of CNSL is still not clear, and therefore, more molecular level-based research studies are needed to clarify the development and treatment of CNSL.

Proteomic technology enables people to observe the development and progression of diseases dynamically and to carry out quantitative analysis. ${ }^{16-18}$ Proteomic analysis of biological fluids has been widely used to screen and identify disease markers. ${ }^{19}$ Recently, various groups have attempted to discover potential protein biomarkers of pediatric leukemia. ${ }^{18-21}$ One study applied surface-enhanced laser desorption/ 
ionization time-of-flight mass spectrometry (SELDI-TOF MS) to analyze the proteomes of cell lysates from childhood leukemia cell lines and childhood leukemia bone marrow samples of different subtypes. ${ }^{22}$ To discover potential protein biomarkers for pediatric ALL, research groups measured serum proteomic profiles between pediatric ALL patients and healthy controls by using SELDI-TOF-MS. In addition, they identified candidate protein biomarkers by using liquid chromatography-tandem mass spectrometry (LC-MS/MS). Notably, the obtained data revealed platelet factor, connective tissue activating peptide III, and two fragments of C3a as potential biomarkers to distinguish pediatric ALL patients from healthy controls and pediatric AML patients. ${ }^{23}$

Cerebrospinal fluid (CSF) analysis provides important and valuable information about process occurring in the CNS. CSF proteomics has been applied to identify biomarkers of various diseases. In normal physiological conditions, 80\% of the protein in the CSF is blood-borne. ${ }^{24,25}$ The change of protein content in CSF provides a new approach to study malignant hematological disease. Researchers conducted quantitative CSF proteomic analysis in newly diagnosed pediatric ALL and lymphoblastic lymphoma (LL) patients. Notably, in this pilot study, the expression of antithrombin III and plasminogen decreased over time in one child who developed CNS thrombosis, compared to other subjects. The results of this study successfully demonstrated the feasibility of CSF quantitative proteomics in ALL and LL. ${ }^{26}$

However, to the best of our knowledge, the CSF proteomic profiling of CNSL has not been reported. In this prospective pilot study, we performed quantitative proteomics by using LC-MS/MS to discover differential expression of CSF protein in newly diagnosed pediatric CNSL patients and healthy controls to discover possible prognostic biomarkers.

\section{Materials and methods}

\section{Sample collection}

The CSF of six pediatric ALL with CNS involvement was obtained from inpatients of Cancer Center, and CSF samples of six controls were obtained from inpatients of pediatrics department, West China Hospital, Sichuan University (2015). All enrolled patients were at the age of 1-11 years. ALL patients with CNS involvement were confirmed by CSF examination. The control group consisted of children with suspected encephalitis, but CSF examination turned out normal. After standard laboratory checks, the CSF samples were centrifugated at $3,000 \times g$ at $4^{\circ} \mathrm{C}$ for 5 minutes to remove the cells, and then stored at $-80^{\circ} \mathrm{C}$ immediately. Our study was approved by the Medical Ethics Committee of West China
Hospital, Sichuan University. All patients' legal guardians signed informed consent, and the study was conducted following the Declaration of Helsinki.

\section{Protein digestion}

After thawed at room temperature, a fixed volume of $30 \mu \mathrm{L}$ CSF was slightly diluted in RIPA buffer $(150 \mathrm{~mm} \mathrm{NaCl}$, $50 \mathrm{~mm}$ Tris- $\mathrm{HCl}$ pH 7.61, NP-40, 1\% deoxycholic acid) with protease and phosphatase inhibitor on ice for $10 \mathrm{~min}$. Then the CSF samples were centrifugated at $2,000 \times g$ at $4^{\circ} \mathrm{C}$ for 5 minutes. After that, the supernatant was determined by Bradford protein assay. Buffer containing $100 \mathrm{mM} \mathrm{NH}_{4} \mathrm{HCO}_{3}$ was added to the centrifuged CSF to create an alkaline environment for trypsin digestion. Then $5 \mathrm{mM}$ D, L-dithiothreitol was added to reduce disulfide bonds for 1 hour at $37^{\circ} \mathrm{C}$, and $55 \mathrm{mM}$ iodoacetamide (IAA) was used to alkylate the cysteines and reacted in the dark for 45 minutes. Finally, $30 \mathrm{mM}$ L-cysteine was needed for the blockade of redundant IAA, and trypsin (Sequencing Grade Modified Trypsin; Promega Corporation, Fitchburg, WI, USA) was added for the digestion of protein samples overnight at a 1:50 (trypsin to protein) ratio and $\mathrm{pH}$ 8.0 at $37^{\circ} \mathrm{C}$. The samples were heated to $95^{\circ} \mathrm{C}$ for inactivating the enzyme, which stopped the digestion reaction. Before the LC-MS/MS analysis, the peptides were desalted by $\mathrm{C} 18$ ZipTip (ZTC18S096; EMD Millipore, Billerica, MA, USA).

\section{LC-MS/MS analysis}

The procedure for LC-MS/MS analysis in the experiment is similar to that in the previous report, with only minor modifications. ${ }^{27}$ All peptide samples were lyophilized and suspended in buffer A ( $2 \%$ acetonitrile, $0.1 \%$ formic acid) before MS/MS. An EASY-nLC 1000 nanoflow LC instrument coupled to a $\mathrm{Q}$ Exactive quadrupole-orbitrap mass spectrometer (Thermo Fisher Scientific, Waltham, MA, USA) was adopted for LC-MS/MS analysis. Magic C18 AQ

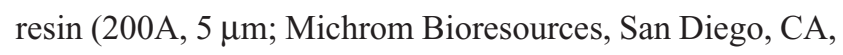
USA) was filled with a $100 \mu \mathrm{m} \times 2 \mathrm{~cm}$ trap column and a $75 \mu \mathrm{m} \times 12 \mathrm{~cm}$ analytical column. The mobile phases were composed of buffer A and buffer B (95\% ACN, 0.1\% formic acid). LC gradient elution conditions initially lasted for 3 minutes for $4 \% \mathrm{~B}$, from 3 to 43 minutes for $22 \% \mathrm{~B}$, followed by 8 minutes for $22 \%-30 \%$, B to $90 \%$ for $52-60$ minutes and lasted for the last 5 minutes at a flow rate of $300 \mathrm{~nL} / \mathrm{min}$. Data-dependent acquisition was conducted in positive ion mode. MS spectra were obtained from 350 to $1,800 \mathrm{~m} / \mathrm{z}$ with a resolution of 70,000 at $\mathrm{m} / \mathrm{z}=200$. The automatic gain control (AGC) value was set to $3 \mathrm{e} 6$ with a maximum fill time of $20 \mathrm{~ms}$. The top 20 most intense parent ions were selected 
with a $1.6 \mathrm{~m} / \mathrm{z}$ isolation window and fragmented normalized collision energy of $27 \%$. The AGC value of MS/MS was set to the target value of $1 \mathrm{e} 6$ with a maximum filling time of $64 \mathrm{~ms}$ and resolution of 17,500. Fragmentation was performed with a high-energy collision induced dissociation collision cell (mass resolution 17,000 at $\mathrm{m} / \mathrm{z}=200$ ). After one repeat count, a dynamic exclusion lasting for 30 seconds was performed. The raw files were acquired using the Q-Exactive plus and then searched and analyzed using Maxquant v1.3. Searches were based on the SwissProt human database. The peptide false discovery rate (FDR) was calculated, and peptide with a value $<1 \%$ FDR was chosen for subsequent data processing.

\section{Statistical analyses}

Multiple comparisons between two groups were performed using a paired $t$-test. $P$-values were calculated based on the Mann-Whitney $U$-test for nonparametric data or two-tailed independent sample $t$-test for parametric data. A $P$-value of $<0.05$ was considered statistically significant.

\section{Proteomic analysis}

Gene Ontology (GO) and REACTOME functional enrichment for differentially expressed genes identified were performed using DAVID 6.8 (https://david-d.ncifcrf.gov/).
Hierarchical clustering in Mev software was utilized for presenting global protein expression. Protein-protein interaction (PPI) network analysis was performed using the online Search Tool for the Retrieval of Interacting Genes database (STRING) (http://string-db.org). Interactions with a combined score $>0.4$ were considered significant. Further, MCODE was used to discover the core module in the PPI network. Finally, GO and REACTOME pathway analyses of proteins in the core module were done online.

\section{Results \\ CSF protein profiles and differential protein expression}

All CSF protein samples were collected from six selected pediatric ALL patients and six healthy pediatric controls in 2015 from pediatrics department of West China Hospital. Age and gender distributions were similar in the two groups. After LC-MS/MS experiments, 455 non-redundant proteins were identified. All the proteins were statistically tested for differential expression. Fifty-one differentially expressed proteins with statistical significance $(P<0.05)$ were found. Among them, 32 proteins were upregulated, and 19 proteins were downregulated. Furthermore, hierarchical clustering was utilized to present protein expression profiles (Figure 1).

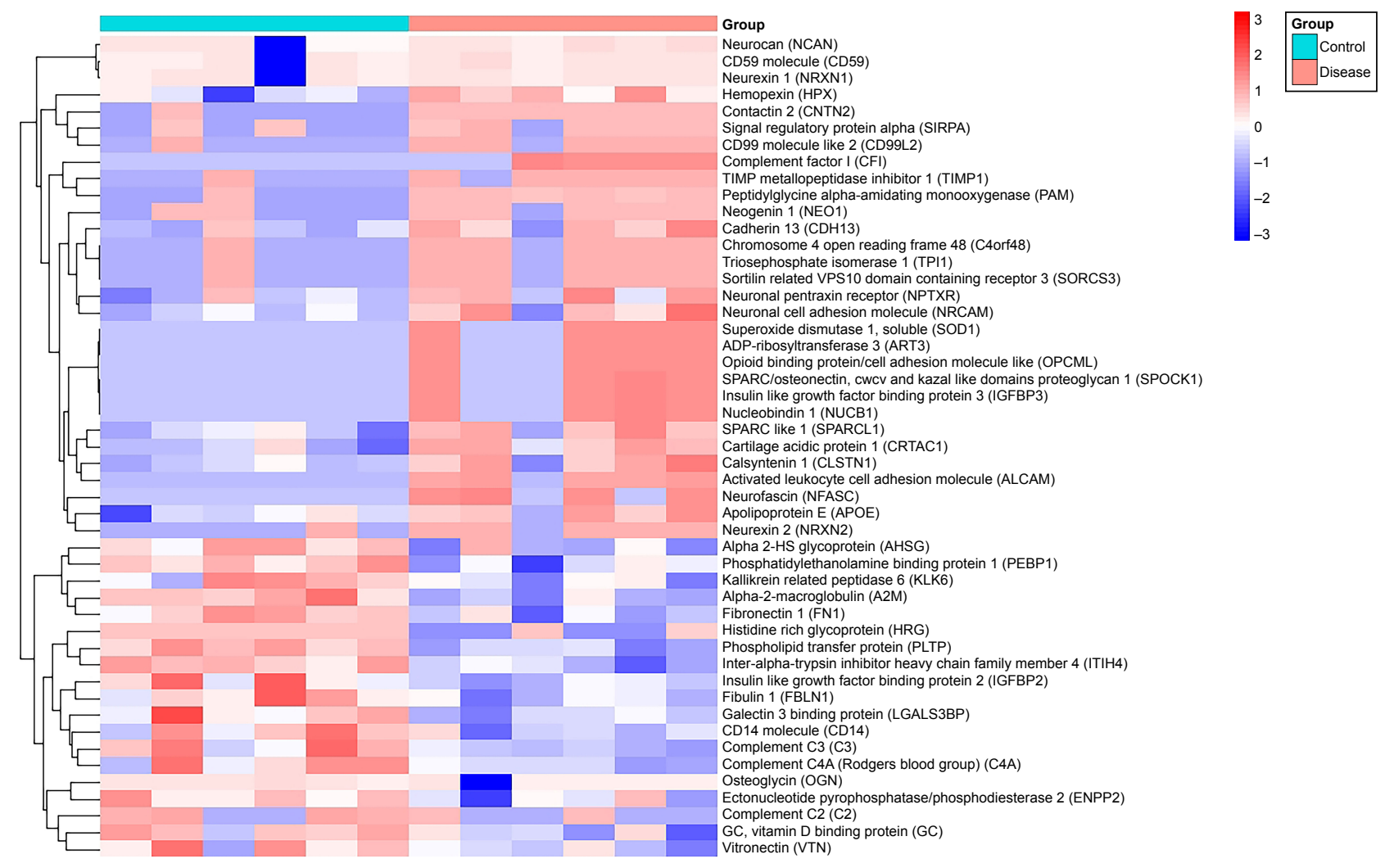

Figure I Hierarchical clustering of global proteins. 
Patients' detailed information were shown in Table S1 and all identified proteins by LC-MS/MS analysis were shown in Table S2.

\section{GO and REACTOME pathway analyses}

To further explore the function of the obtained proteins, GO and REACTOME pathway analyses were performed. A total of 49 gene IDs were found in DAVID. The results of the GO analysis showed that 47 differentially expressed genes were related to 57 terms on the category of "Biological Process." Among biological processes, the overrepresented terms include cell adhesion (13 genes, $P=2.53 \mathrm{e}-09$ ), negative regulation of endopeptidase activity (10 genes, $P=3.41 \mathrm{e}-11$ ), platelet degranulation ( 8 genes, $P=1.17 \mathrm{e}-08$ ), signal transduction ( 8 genes, $P=0.0373$ ), receptor-mediated endocytosis ( 7 genes, $P=1.10 \mathrm{e}-05$ ), regulation of complement activation (6 genes, $P=1.67 \mathrm{e}-08$ ), CNS development (5 genes, $P=3.21 \mathrm{e}-04$ ), regulation of cell growth (4 genes, $P=0.0014)$, complement activation, classical pathway (4 genes, $P=0.0025$ ), and axon guidance (4 genes, $P=0.0094$ ). The results indicated that platelet degranulation, regulation of immunity, cell growth, and CNS might play an important role in the development of pediatric ALL. Top ten biological processes obtained from GO analysis of all differentially expressed proteins were shown on Figure 2A.
As REACTOME pathway analysis revealed, the differentially expressed genes were associated with a total of 189 pathways. The most representative pathways were immune system (17 genes, $P=0.0419$ ), post-translational protein modification (12 genes, $P=0.0241$ ), regulation of insulinlike growth factor (IGF) transport and uptake by insulinlike growth factor binding proteins (IGFBPs) (10 genes, $P=1.55 \mathrm{e}-10)$, hemostasis (10 genes, $P=0.0017)$, innate immune system (10 genes, $P=0.042$ ), post-translational protein phosphorylation ( 9 genes, $P=9.17 \mathrm{e}-10$ ), platelet degranulation ( 8 genes, $P=1.19 \mathrm{e}-7$ ), response to elevated platelet cytosolic $\mathrm{Ca}^{+}$( 8 genes, $\left.P=1.73 \mathrm{e}^{-7}\right)$, platelet activation, signaling, and aggregation ( 8 genes, $P=4.15 \mathrm{e}-5$ ). The results indicated that the immune system, cell growth, and platelet function might be essential for the development of pediatric ALL. Top ten pathways obtained from REACTOME pathway analysis of all differentially expressed proteins are shown on Figure 2B.

\section{PPI network analysis}

To examine the interaction between these differentially expressed genes, we used the STRING database to perform PPI network analysis. The results revealed that an interaction network comprising 31 genes and 64 gene interaction relations was found (Figure 3C).
A

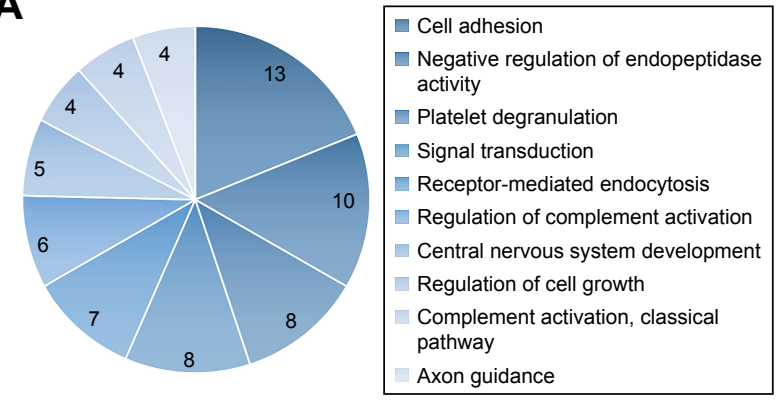

C

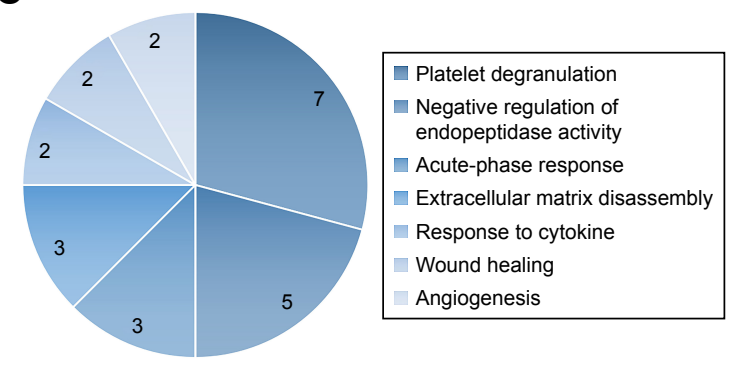

B

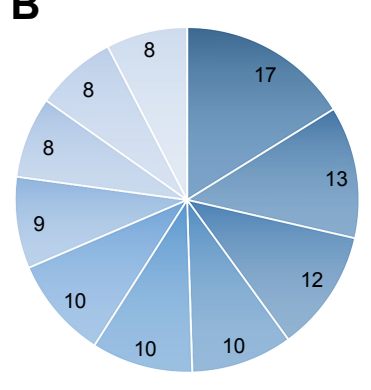

Immune system

- Metabolism of proteins

- Post-translational protein modification

- Regulation of insulin-like growth factor (IGF) transport and uptake by insulin-like growth factor-binding proteins (IGFBPs)

- Hemostasis

- Innate immune system

- Post-translational protein phosphorylation

- Platelet degranulation

Response to elevated platelet cytosolic $\mathrm{Ca}^{+}$

Platelet activation, signaling and aggregation

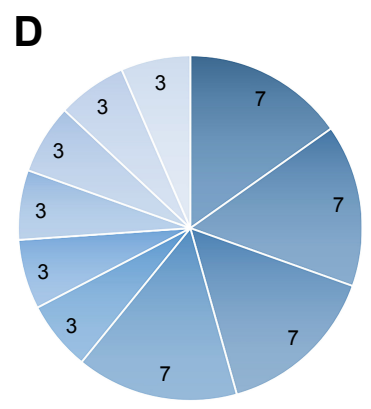

Figure 2 Gene Ontology (GO) and REACTOME pathway analysis of quantified proteins. (A) Top ten biological processes obtained from GO analysis of all differentially expressed proteins. (B) Top ten pathways obtained from REACTOME pathway analysis of all differentially expressed proteins. (C) Biological processes obtained from GO analysis of cluster I proteins. (D) Top ten pathways obtained from REACTOME pathway analysis of cluster I proteins. 
A

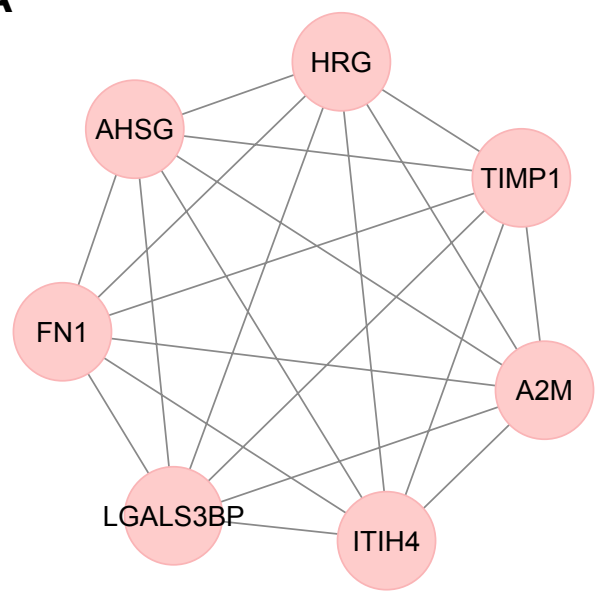

B

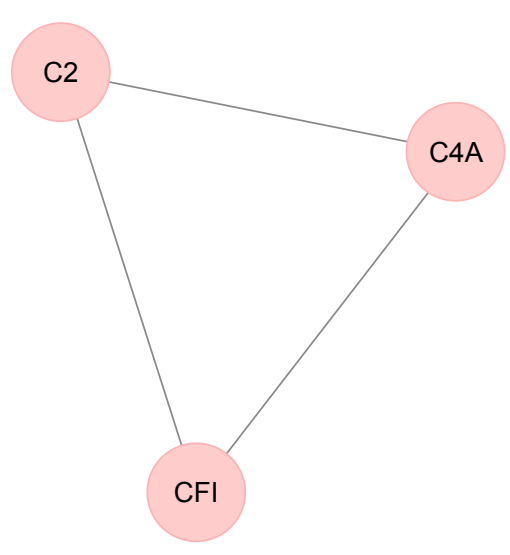

C

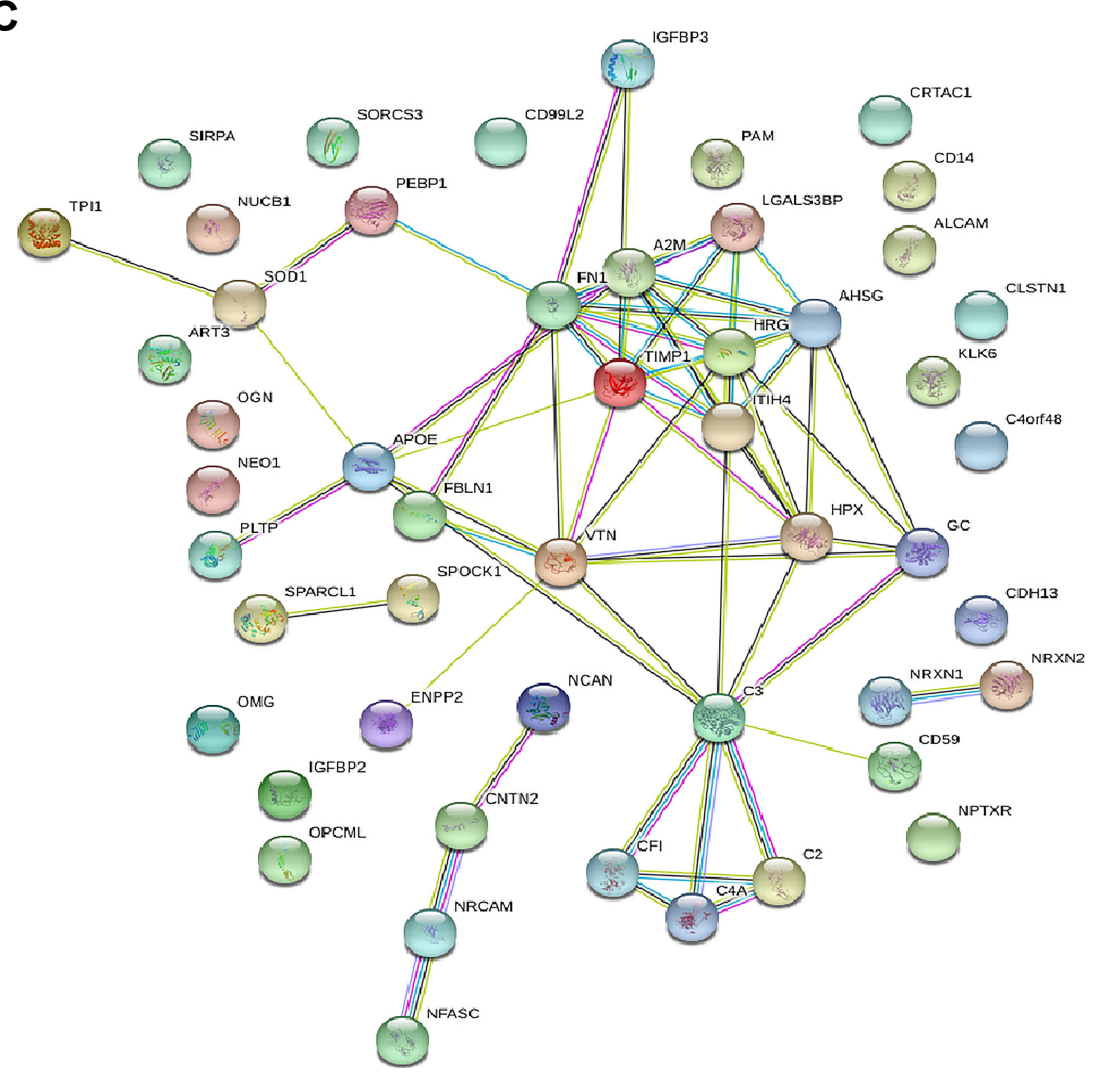

Figure 3 Protein-protein interaction (PPI) and MCODE analysis of differentially expressed proteins. (A) Proteins and interactions of cluster I identified by MCODE analysis. (B) Proteins and interactions of cluster 2 identified by MCODE analysis. (C) Proteins and interactions of PPI network.

Further, we used MCODE to discover core module in the PPI network. The results showed that two core clusters including 10 genes were found. Cluster 1 contained seven genes with 21 gene interaction relations (MCODE score $=5.25$, node $=7$, edges $=21$ ). Proteins involved in cluster 1 were LGALS3BP, TIMP1, A2M, AHSG, FN1, HRG, and ITIH4 (Figure 3A). Cluster 2 included 3 genes with 3 interaction relations $(\mathrm{MCODE}$ score $=1.5$, node $=3$, edges $=3$ ). Proteins involved in cluster 2 were CF I, C2, and C4A (Figure 3B).

\section{GO function and REACTOME pathway analysis of cluster proteins}

For cluster 1 proteins, GO analysis revealed that seven terms were on the category of "Biological Process" (Figure 2C). Platelet degranulation ( 7 genes, $P=4.60 \mathrm{e}^{-14}$ ), negative regulation of endopeptidase activity (5 genes, $P=3.81 \mathrm{e}-8$ ), acute-phase response ( 3 genes, $P=7.84 \mathrm{e}-5$ ), and extracellular matrix disassembly ( 3 genes, $3.00 \mathrm{e}-4)$ were the most representative terms. REACTOME pathway analysis showed that 
a total of 57 pathways were involved, with platelet degranulation ( 7 genes, $P=2.82 \mathrm{e}-14$ ), response to elevated platelet cytosolic $\mathrm{Ca}^{+}$( 7 genes, $P=3.70 \mathrm{e}^{-14}$ ), platelet activation, signaling and aggregation ( 7 genes, $P=6.60 \mathrm{e}^{-12}$ ), hemostasis ( 7 genes, $P=6.27 \mathrm{e}^{-9}$ ) as the most predominant. Notably, results of cluster 1 proteins were highly consistent with the results of all the differentially expressed proteins. Top 10 pathways obtained from REACTOME pathway analysis of cluster 1 proteins are shown in Figure 2D.

Regarding cluster 2, CF I, C2, and C4A were all related to immune system especially the activity of complement according to GO and REACTOME pathway analysis.

\section{Discussion}

ALL is the main type of leukemia that causes CNSL. ${ }^{28}$ The incidence of CNSL in pediatric ALL patients is much higher than that in adults. ${ }^{28,29} \mathrm{CNSL}$ is a special extramedullary infiltrating form of leukemia cells that invade the CNS. ${ }^{30}$ Leukemia cells may enter the CNS (including the meninges) through direct dissemination or blood circulation. ${ }^{29-31}$ Few studies have investigated the molecular mechanisms and pathways for CNSL. Currently, a mechanism of ALL cell entry into the CNS by targeting VEGF signaling has been identified by animal model. ${ }^{32}$ However, the results still need more experiments for validation. In addition, irreversible damages to the CNS remain one of the major causes of ALL treatment failure. ${ }^{29}$ Once CNS infiltration occurs, it is extremely difficult to control with poor clinical outcomes. Therefore, there is an urgent demand to better understand the pathogenesis of CNSL and identify potential protein markers in the diagnosis and treatment of ALL with CNS infiltration.

CSF is an excellent indicator of brain activity and processes. $^{33}$ There is also a consensus that CSF is one of the most suitable proximal fluids for analysis of biomarkers in neurological disorders. ${ }^{34,35}$ It contains normal and diseaserelated protein components which provide insight into the function of the brain. By analyzing the proteomics of the collected CSF, we were able to identify proteins that were significantly changed in ALL patients with CNS infiltration. In our current study, CSF protein samples were collected from six selected pediatric ALL patients and six control groups. Using LC-MS/MS, 51 proteins were identified to be significantly different between the two groups $(P<0.05)$ including 32 proteins upregulated and 19 proteins downregulated. Out of these 51 genes, 49 were found in DAVID. Using GO analysis, 47 differentially expressed genes were related to 57 terms in the biological processes category. The results showed that the differentially expressed proteins were mainly grouped in platelet degranulation, regulation of immunity, cell growth, and CNS.

To better understand the interaction between these differentially expressed proteins, we performed PPI analysis. Two different clusters were identified as shown in Figure 3. These results indicate that the proteins we have identified were not random; rather they have intricate interaction networks. More specifically, cluster 1 proteins including TIMP1, LGALS3BP, A2M, AHSG, FN1, HRG, and ITIH4 were all related to cancer. ${ }^{36-61}$ Cluster 2 proteins including $\mathrm{CF}$ I, C2, and $\mathrm{C} 4 \mathrm{~A}$ were all related to immune system especially the activity of complement. ${ }^{62-70}$

TIMP1 is a glycoprotein that inhibits the proteolytic activity of matrix metalloproteinases in the extracellular space. ${ }^{36}$ Previous studies have reported that TIMP1 had a positive effect on tumor metastases and progression inhibition. ${ }^{37,38}$ However, new pieces of evidence have indicated that the overexpression of TIMP1 was associated with poor prognosis in several tumors such as non-Hodgkin lymphomas, ${ }^{39}$ breast cancer, ${ }^{40,41}$ colorectal cancer, and papillary thyroid cancer. ${ }^{41}$ Recently, increased TIMP1 expression has been associated with unfavorable clinical outcomes in children with ALL. The proposed mechanism is the anti-apoptotic function in different cancer cell lines of TIMP1 by activating the FAK/Akt axis and the Bcl-2 survival signaling pathway. ${ }^{42}$ In the present study, higher levels of TIMP1 expression were also detected. Therefore, we assumed TIMP1 might be a key factor involved in the pathogenesis of CNSL.

LGALS3BP is also known as galectin-3-binding protein, which modulates cell-cell and cell-matrix interactions. ${ }^{43}$ LGALS3BP has been found to have a complex function in the development of human cancers. High LGALS3BP expression has been implicated with the presence of metastasis in some solid tumors such as non-small-cell lung cancer ${ }^{43,44}$ and breast cancer. ${ }^{43}$ However, one study reported that the overexpression of the protein in a series of tumor-derived cell lines reduced tumorigenic potential in athymic mice. ${ }^{45}$ To the best of our knowledge, literature evidence associated with ALL is limited. Further studies are needed to investigate the role of LGALS3BP in tumors.

As a protease inhibitor and cytokine transporter, alpha2-macroglobulin (A2M) was associated with inflammation and cancer. ${ }^{46,47}$ Previous study has reported that A2M was identified as a candidate biomarker for the diagnosis of B-ALL by serum proteomic analysis. ${ }^{48}$ In addition, altered expression of A2M and FN1 (fibronectin 1) showed 
discriminating signatures in breast cancer subtypes. ${ }^{49}$ FN1 is a glycoprotein present in the extracellular matrix and at the cell surface, which plays an important role in cancer progression by promoting cell adhesion and migration. ${ }^{50,51} \mathrm{In}$ the present study, A2M and FN1 were also found to be differentially expressed between the two groups which might be of potential diagnostic and prognostic significance for CNSL.

AHSG is a glycoprotein produced by hepatocytes, which has been reported to be downregulated in serum in certain solid tumors, as well as hematological malignancies including acute lymphocytic, acute nonlymphocytic, chronic granulocytic, and chronic myelomonocytic leukemias..$^{52,53}$ The reduction of AHSG is mainly considered to be caused by the infiltration of liver and increased consumption..$^{52}$ Our study found that the concentration of AHSG in CSF decreased in ALL patients as compared to that in healthy controls. In addition, AHSG is found to be involved in brain development, and it may function as a transforming growth factor- $\beta$ (TGF- $\beta$ ) receptor analog. ${ }^{54}$ It is known that TFG- $\beta$ regulates a wide range of physiological and pathological processes in the brain including brain inflammatory response and extracellular matrix regulation. ${ }^{55}$ Therefore, the change of AHSG concentration might be a useful laboratory parameter for the assessment of ALL clinical status, especially for CNSL.

Histidine-rich glycoprotein (HRG) is an abundant glycoprotein with multidomain structure. ${ }^{56}$ It modulates various biological processes including cell adhesion, immunity, angiogenesis, and coagulation, most of which involved in tumor metastasis and progression. Studies have suggested that HRG suppressed tumor metastasis and growth by promoting antitumor immune response and tumor vasculature dysfunction. ${ }^{57}$ In vivo, HRG was demonstrated to control tumor metastasis in mouse models of ductal adenocarcinoma, insulinoma, and fibrosarcoma. ${ }^{56-58}$ In the present study, the level of HRG decreased, which is consistent with the lowered expression of HRG in ovarian cancer and liver cancer. Based on this, we wondered HRG might be crucial for CNSL development.

ITIH4 is a glycoprotein secreted by liver in blood with the main function of regulating extracellular matrix. ${ }^{59} \mathrm{It}$ has been found to be involved in genesis, metastasis, and development of several solid tumors. ${ }^{60}$ Research groups have observed the dynamic change of serum ITIH4 concentration in the developmental progress of HCC. ${ }^{61}$ It is confirmed that the decreased ITIH4 concentration was related to the genesis of HCC and high HCC risk. ${ }^{61}$ Declined ITIH4 expression was also detected in the present study. Hence, ITIH4 might serve as a warning marker of CNSL genesis.
The complement system consists of more than 30 soluble proteins and membrane binding proteins, which are widely involved in the body's anti-microbial defense reaction and immune regulation, and can also mediate the damage response of immunopathology. ${ }^{62}$ The complement system has three activation pathways and eventually forms membrane attack complex (MAC). MAC can dissolve tumor cells and therefore inhibit tumor progress. ${ }^{63}$ Complement component 2 (C2) is involved in the classical and lectin pathway of complement activation, and it is required for the formation of MAC. ${ }^{64}$ Complement activation product $\mathrm{C} 4 \mathrm{a}$ can stimulate inflammatory reaction to eliminate pathogenic microorganisms. ${ }^{65}$ In the present study, the concentrations of $\mathrm{C} 2$ and $\mathrm{C} 4 \mathrm{a}$ in $\mathrm{CSF}$ were decreased. The underlying mechanism might be the activation of the immune system by tumor cells leading to an increase in $\mathrm{C} 2$ and decrease in $\mathrm{C} 4 \mathrm{a}$.

Complement factor I (CFI) is of vital importance in the regulation of complement activation by cleaving $\mathrm{C} 3 \mathrm{~b}$ and $\mathrm{C} 4 \mathrm{~b}$ with cofactors complement receptor I (CD35), membrane cofactor protein (CD46), and complement factor $\mathrm{H}$ (CFH) ${ }^{66}$ Expression of CFI has been observed in breast cancer cells, ${ }^{67}$ NSCSC cells,${ }^{68}$ cutaneous squamous cell carcinoma (cSCC) cells, ${ }^{69}$ and glioma cells. ${ }^{70}$ Current evidence suggested that CFI had an important role in promoting cSCC growth and invasion, possibly by using complement inhibitors for immune escape. ${ }^{69}$ In addition, high CFI expression has been reported to be positively correlated with breast cancer tumor size and associated with poor clinical outcomes ${ }^{67}$ In the present study, we observed an elevated concentration of CFI. Therefore, CFI may participate in the development of CNSL and be a valuable indicator of CNSL diagnosis.

We have a few limitations in our current study. First, we have a relatively small sample size, so results showed in this study may or may not be a good representative of the whole. However, the 12 samples (2 groups of 6 ) that we obtained were relatively consistent in terms of analysis which gave us some confidence in our results. In addition, our results are consistent with published results in the literature. ${ }^{42,52,56,61,69}$ Next, the goal of the current study is a pilot study, so the depth of is not great. But publishing our pilot results will allow other groups to analyze some of these specific targets in depth. We are also analyzing some of these proteins more specifically and in detail. Moreover, due to ethical concerns and difficulties in collecting pediatric samples, our study lacked the distinction between ALL with and without CNS involvement. But our study design was in accordance with 
previous high-quality studies on CSF research, which was relatively rigorous. Finally, all our samples came from the Chinese Han population, which may or may not be a good representative for other ethnic groups. Due to the limitation to obtaining samples, Chinese Han population were the only samples that were available in our regions.

\section{Conclusion}

We have used CSF samples from six ALL patients and six normal individuals and discovered significant protein changes which may aid as potential biomarkers in the diagnosis and prognosis of childhood ALL with CNS infiltration. We hope our pilot study can provide an excellent foundation for the future investigators to further analyze these changes in proteins and identify their potential role in the etiology of ALL with CNS infiltration.

\section{Author contributions}

All authors contributed to data analysis, drafting and revising the article, gave final approval of the version to be published, and agree to be accountable for all aspects of the work.

\section{Disclosure}

The authors report no conflicts of interest in this work.

\section{References}

1. Dores GM, Devesa SS, Curtis RE, Linet MS, Morton LM. Acute leukemia incidence and patient survival among children and adults in the United States, 2001-2007. Blood. 2012;119(1):34-43. doi:10.1182/ blood-2011-04-347872

2. Hunger SP, Mullighan CG. Acute lymphoblastic leukemia in children. NEngl JMed. 2015;373(16):1541-1552. doi:10.1056/NEJMra1400972

3. Ampatzidou M, Panagiotou JP, Paterakis G, et al. Childhood acute lymphoblastic leukemia: 12 years of experience, using a Berlin-FrankfurtMunster approach, in a Greek center. Leuk Lymphoma. 2015;56(1): 251-255. doi:10.3109/10428194.2014.916801

4. Bhojwani D, Yang JJ, Pui CH. Biology of childhood acute lymphoblastic leukemia. Pediatr Clin North Am. 2015;62(1):47-60. doi:10.1016/j.pcl.2014.09.004

5. Jatczak-Gaca A, Styczynski J, Koltan A, Debski R, Pogorzala M, Wysocki M. Results of therapy in children with acute lymphoblastic leukemia in over 50 years of experience in a single center in Poland. Leuk Lymphoma. 2015;56(7):2212-2214. doi:10.3109/10428194.2014 .999325

6. Tsurusawa M. [Childhood acute lymphoblastic leukemia; pathophysiology and treatment strategy]. Rinsho Ketsueki. 2013;54(11):2038-2046.

7. Belgaumi AF, Al-Seraihy A, Siddiqui KS, et al. Outcome of risk adapted therapy for relapsed/refractory acute lymphoblastic leukemia in children. Leuk Lymphoma. 2013;54(3):547-554. doi:10.3109/10428194.2012.71 9616

8. Bhojwani D, Pui CH. Relapsed childhood acute lymphoblastic leukaemia. Lancet Oncol. 2013;14(6):70580-70586. doi:10.1016/S1470-2045 (12)70580-6

9. Baytan B, Evim MS, Guler S, Gunes AM, Okan M. Acute central nervous system complications in pediatric acute lymphoblastic leukemia. Pediatr Neurol. 2015;53(4):312-318. doi:10.1016/j.pediatrneurol. 2015.03.006
10. Krull KR, Hockenberry MJ, Miketova P, Carey M, Moore IM. Chemotherapy-related changes in central nervous system phospholipids and neurocognitive function in childhood acute lymphoblastic leukemia. Leuk Lymphoma. 2013;54(3):535-540. doi:10.3109/10428194.2012. 717080

11. Manley S, Keenan R, Campbell H, Caswell M, Pizer B. No evidence for routine cerebrospinal fluid cytology in detecting asymptomatic central nervous system relapse in children with acute lymphoblastic leukaemia: 20 years' experience of a UK primary treatment centre. Br J Haematol. 2014;164(3):462-464. doi:10.1111/bjh.12646

12. Zajac-Spychala O, Wachowiak J. [Late sequelae of central nervous system prophylaxis in children with acute lymphoblastic leukemia: high doses of intravenous methotrexate versus radiotherapy of the central nervous system-review of literature]. Med Wieku Rozwoj. 2012;16(2):128-137.

13. Gorshein E, Kalathil S, Gharibo M. Prolonged survival of acute lymphoblastic leukemia with intrathecal treatments for isolated central nervous system relapse. Case Rep Hematol. 2018;31:8765285.

14. Marz M, Meyer S, Erb U, et al. Pediatric acute lymphoblastic leukemiaConquering the CNS across the choroid plexus. Leuk Res. 2018;71: 47-54. doi:10.1016/j.leukres.2018.07.001

15. Yao H, Price TT, Cantelli G, et al. Leukaemia hijacks a neural mechanism to invade the central nervous system. Nature. 2018;560(7716): 55-60. doi:10.1038/s41586-018-0342-5

16. Lopez Villar E, Wu D, Cho WC, Madero L, Wang X. Proteomics-based discovery of biomarkers for paediatric acute lymphoblastic leukaemia: challenges and opportunities. J Cell Mol Med. 2014;18(7):1239-1246. doi: $10.1111 /$ jcmm. 12319

17. Jiang N, Kham SK, Koh GS, et al. Identification of prognostic protein biomarkers in childhood acute lymphoblastic leukemia (ALL). J Proteomics. 2011;74(6):843-857. doi:10.1016/j.jprot.2011.02.034

18. Wang D, Lv YQ, Liu YF, Du XJ, Li B. Differential protein analysis of lymphocytes between children with acute lymphoblastic leukemia and healthy children. Leuk Lymphoma. 2013;54(2):381-386. doi:10.3 109/10428194.2012.713104

19. Xu Y, Zhuo J, Duan Y, et al. Construction of protein profile classification model and screening of proteomic signature of acute leukemia. Int J Clin Exp Pathol. 2014;7(9):5569-5581.

20. Braoudaki M, Lambrou GI, Vougas K, Karamolegou K, Tsangaris GT, Tzortzatou-Stathopoulou F. Protein biomarkers distinguish between high- and low-risk pediatric acute lymphoblastic leukemia in a tissue specific manner. J Hematol Oncol. 2013;6(52):1756-8722. doi:10.1186/ 1756-8722-6-52

21. Wang D, Lu YQ, Liu YF, Su SF, Li B. [Identification of new markers for childhood acute lymphoblastic leukemia by MALDI-TOF-MS]. Zhongguo Shi Yan Xue Ye Xue Za Zhi. 2012;20(6):1365-1369.

22. Hegedus CM, Gunn L, Skibola CF, et al. Proteomic analysis of childhood leukemia. Leukemia. 2005;19(10):1713-1718. doi:10.1038/ sj.leu.2403897

23. Shi L, Zhang J, Wu P, et al. Discovery and identification of potential biomarkers of pediatric acute lymphoblastic leukemia. Proteome Sci. 2009;7(7):1477-5956. doi:10.1186/1477-5956-7-7

24. Galicia N, Diez P, Degano RM, Guest PC, Ibarrola N, Fuentes M. Proteomic biomarker identification in cerebrospinal fluid for leptomeningeal metastases with neurological complications. Adv Exp Med Biol. 2017;974:85-96. doi:10.1007/978-3-319-52479-5_5

25. Roy S, Josephson SA, Fridlyand J, et al. Protein biomarker identification in the CSF of patients with CNS lymphoma. J Clin Oncol. 2008;26(1):96-105. doi:10.1200/JCO.2007.12.1053

26. Priola GM, Foster MW, Deal AM, Richardson BM, Thompson JW, Blatt J. Cerebrospinal fluid proteomics in children during induction for acute lymphoblastic leukemia: A pilot study. Pediatr Blood Cancer. 2015;62(7):1190-1194. doi:10.1002/pbc.25420

27. Gong $\mathrm{Y}, \mathrm{Pu} \mathrm{W}$, Jin $\mathrm{H}$, et al. Quantitative proteomics of CSF reveals potential predicted biomarkers for extranodal NK-/T-cell lymphoma of nasal-type with ethmoidal sinus metastasis. Life Sci. 2018;198:94-98. doi:10.1016/j.1fs.2018.02.035 
28. Zhou W, Li JM. [Mechanism and early evaluation of CNS infiltration in acute lymphocytic leukemia-review]. Zhongguo Shi Yan Xue Ye Xue Za Zhi.2013;21(5):1361-1364. doi:10.7534/j.issn.1009-2137.2013.05.055

29. Levinsen M, Taskinen M, Abrahamsson J, et al; Nordic Society of Paediatric Haematology and Oncology (NOPHO). Clinical features and early treatment response of central nervous system involvement in childhood acute lymphoblastic leukemia. Pediatr Blood Cancer. 2014;61(8):1416-1421. doi:10.1002/pbc.24981

30. Gassas A, Krueger J, Alvi S, Sung L, Hitzler J, Lieberman L. Diagnosis of central nervous system relapse of pediatric acute lymphoblastic leukemia: impact of routine cytological CSF analysis at the time of intrathecal chemotherapy. Pediatr Blood Cancer. 2014;61(12):2215-2217. doi:10.1002/pbc. 25235

31. Frishman-Levy L, Shemesh A, Bar-Sinai A, et al. Central nervous system acute lymphoblastic leukemia: role of natural killer cells. Blood. 2015;125(22):3420-3431. doi:10.1182/blood-2014-08-595108

32. Munch V, Trentin L, Herzig J, et al. Central nervous system involvement in acute lymphoblastic leukemia is mediated by vascular endothelial growth factor. Blood. 2017;130(5):643-654. doi:10.1182/blood-2017-03-769315

33. Crespo-Solis E, Lopez-Karpovitch X, Higuera J, Vega-Ramos B. Diagnosis of acute leukemia in cerebrospinal fluid (CSF-acute leukemia). Curr Oncol Rep . 2012;14(5):369-378. doi:10.1007/s11912-012-0248-6

34. Sirvent N, Suciu S, Rialland X, et al. Prognostic significance of the initial cerebro-spinal fluid (CSF) involvement of children with acute lymphoblastic leukaemia (ALL) treated without cranial irradiation: results of European Organization for Research and Treatment of Cancer (EORTC) Children Leukemia Group study 58881. Eur J Cancer. 2011; 47(2):239-247. doi:10.1016/j.ejca.2010.10.019

35. Winick N, Devidas M, Chen S, et al. Impact of initial CSF findings on outcome among patients with national cancer institute standardand high-risk B-cell acute lymphoblastic leukemia: a report from the children's oncology group. J Clin Oncol. 2017;35(22):2527-2534. doi:10.1200/JCO.2016.71.4774

36. Suminoe A, Matsuzaki A, Hattori H, Koga Y, Ishii E, Hara T. Expression of matrix metalloproteinase (MMP) and tissue inhibitor of MMP (TIMP) genes in blasts of infant acute lymphoblastic leukemia with organ involvement. Leuk Res. 2007;31(10):1437-1440. doi:10.1016/j. leukres.2007.01.015

37. Luparello C, Avanzato G, Carella C, Pucci-Minafra I. Tissue inhibitor of metalloprotease (TIMP)-1 and proliferative behaviour of clonal breast cancer cells. Breast Cancer Res Treat. 1999;54(3):235-244.

38. Guedez L, Stetler-Stevenson WG, Wolff L, et al. In vitro suppression of programmed cell death of B cells by tissue inhibitor of metalloproteinases-1. J Clin Invest. 1998;102(11):2002-2010. doi:10.1172/JCI2881

39. Kossakowska AE, Urbanski SJ, Edwards DR. Tissue inhibitor of metalloproteinases-1 (TIMP-1) RNA is expressed at elevated levels in malignant non-Hodgkin's lymphomas. Blood. 1991;77(11): 2475-2481.

40. Wurtz SO, Schrohl AS, Mouridsen H, Brunner N. TIMP-1 as a tumor marker in breast cancer-an update. Acta Oncol. 2008;47(4):580-590. doi:10.1080/02841860802022976

41. Hawthorn L, Stein L, Varma R, Wiseman S, Loree T, Tan D. TIMP1 and SERPIN-A overexpression and TFF3 and CRABP1 underexpression as biomarkers for papillary thyroid carcinoma. Head Neck 2004;26(12):1069-1083. doi:10.1002/hed.20099

42. Scrideli CA, Cortez MA, Yunes JA, et al. mRNA expression of matrix metalloproteinases (MMPs) 2 and 9 and tissue inhibitor of matrix metalloproteinases (TIMPs) 1 and 2 in childhood acute lymphoblastic leukemia: potential role of TIMP1 as an adverse prognostic factor. Leuk Res. 2010;34(1):32-37. doi:10.1016/j.leukres.2009.10.007

43. Grassadonia A, Tinari N, Iurisci I, et al. $90 \mathrm{~K}$ (Mac-2 BP) and galectins in tumor progression and metastasis. Glycoconj J. 2002;19(7-9):551-556. doi:10.1023/B:GLYC.0000014085.00706.d4

44. Marchetti A, Tinari N, Buttitta F, et al. Expression of $90 \mathrm{~K}$ (Mac-2 BP) correlates with distant metastasis and predicts survival in stage I non-small cell lung cancer patients. Cancer Res. 2002;62(9): 2535-2539.
45. Jallal B, Powell J, Zachwieja J, et al. Suppression of tumor growth in vivo by local and systemic $90 \mathrm{~K}$ level increase. Cancer Res. 1995; 55(15):3223-3227.

46. Acuner-Ozbabacan ES, Engin BH, Guven-Maiorov E, et al. The structural network of Interleukin-10 and its implications in inflammation and cancer. BMC Genomics. 2014;15(Suppl 4):S2. doi:10.1186/ 1471-2164-15-S4-S2

47. Ganter U, Strauss S, Jonas U, et al. Alpha 2-macroglobulin synthesis in interleukin-6-stimulated human neuronal (SH-SY5Y neuroblastoma) cells. Potential significance for the processing of Alzheimer betaamyloid precursor protein. FEBS Lett. 1991;282(1):127-131.

48. Cavalcante MDES, Torres-Romero JC, Lobo MD, et al. A panel of glycoproteins as candidate biomarkers for early diagnosis and treatment evaluation of B-cell acute lymphoblastic leukemia. Biomark Res. 2016;4:1. doi:10.1186/s40364-016-0055-6

49. Suman S, Basak T, Gupta P, et al. Quantitative proteomics revealed novel proteins associated with molecular subtypes of breast cancer. J Proteomics. 2016;148:183-193. doi:10.1016/j.jprot.2016.07.033

50. Bae YK, Kim A, Kim MK, Choi JE, Kang SH, Lee SJ. Fibronectin expression in carcinoma cells correlates with tumor aggressiveness and poor clinical outcome in patients with invasive breast cancer. Hum Pathol. 2013;44(10):2028-2037. doi:10.1016/j.humpath.2013.03.006

51. Fernandez-Garcia B, Eiró N, Marin L, et al. Expression and prognostic significance of fibronectin and matrix metalloproteases in breast cancer metastasis. Histopathology. 2014;64(4):512-522. doi:10.1111/ his. 12300

52. Kalabay L, Cseh K, Benedek S, et al. Serum alpha 2-HS glycoprotein concentration in patients with hematological malignancies. A follow-up study. Ann Hematol. 1991;63(5):264-269.

53. Triffitt JT, Gebauer U, Ashton BA, Owen ME, Reynolds JJ. Origin of plasma alpha2HS-glycoprotein and its accumulation in bone. Nature. 1976;262(5565):226-227.

54. Demetriou M, Binkert C, Sukhu B, Tenenbaum HC, Dennis JW. Fetuin/alpha2-HS glycoprotein is a transforming growth factor-beta type II receptor mimic and cytokine antagonist. J Biol Chem. 1996; 271(22):12755-12761

55. Masliah E, Ho G, Wyss-Coray T. Functional role of TGF beta in Alzheimer's disease microvascular injury: lessons from transgenic mice. Neurochem Int. 2001;39(5-6):393-400.

56. Tugues S, Honjo S, Konig C, et al. Genetic deficiency in plasma protein HRG enhances tumor growth and metastasis by exacerbating immune escape and vessel abnormalization. Cancer Res. 2012;72(8):1953-1963. doi:10.1158/0008-5472.CAN-11-2194

57. Rolny C, Mazzone M, Tugues S, et al. HRG inhibits tumor growth and metastasis by inducing macrophage polarization and vessel normalization through downregulation of PlGF. Cancer Cell. 2011;19(1):31-44. doi:10.1016/j.ccr.2010.11.009

58. Cedervall J, Zhang Y, Ringvall M, et al. HRG regulates tumor progression, epithelial to mesenchymal transition and metastasis via platelet-induced signaling in the pre-tumorigenic microenvironment. Angiogenesis. 2013;16(4):889-902. doi:10.1007/s10456-013-9363-8

59. van Den Broek I, Sparidans RW, van Winden AW, et al. The absolute quantification of eight inter-alpha-trypsin inhibitor heavy chain 4 (ITIH4)-derived peptides in serum from breast cancer patients. Proteomics Clin Appl. 2010;4(12):931-939. doi:10.1002/prca.201000035

60. Lee EJ, Yang SH, Kim KJ, et al. Inter-alpha inhibitor H4 as a potential biomarker predicting the treatment outcomes in patients with hepatocellular carcinoma. Cancer Res Treat. 2018;50(3):646-657. doi:10.4143/ crt.2016.550

61. Li X, Li B, Li B, et al. ITIH4: effective serum marker, early warning and diagnosis, hepatocellular carcinoma. Pathol Oncol Res. 2018; 24(3):663-670. doi:10.1007/s12253-017-0285-4

62. Richie E, Culbert S, Sullivan MP, Van Eys J. Acute lymphoblastic leukemia and complement receptors. Blood. 1978;52(2):467.

63. Tegla CA, Cudrici C, Patel S, et al. Membrane attack by complement: the assembly and biology of terminal complement complexes. Immunol Res. 2011;51(1):45-60. doi:10.1007/s12026-011-8239-5 
64. Klaska I, Nowak JZ. [The role of complement in physiology and pathology]. Postepy Hig Med Dosw (Online). 2007;61:167-177.

65. Wang H, Ricklin D, Lambris JD. Complement-activation fragment C4a mediates effector functions by binding as untethered agonist to proteaseactivated receptors 1 and 4. Proc Natl Acad Sci U S A. 2017;114(41): 10948-10953. doi:10.1073/pnas.1707364114

66. Nilsson SC, Sim RB, Lea SM, Fremeaux-Bacchi V, Blom AM. Complement factor I in health and disease. Mol Immunol. 2011;48(14): 1611-1620. doi:10.1016/j.molimm.2011.04.004

67. Okroj M, Holmquist E, Nilsson E, Anagnostaki L, Jirstrom K, Blom AM. Local expression of complement factor I in breast cancer cells correlates with poor survival and recurrence. Cancer Immunol Immunother. 2015;64(4):467-478. doi:10.1007/s00262-015-1658-8
68. Okroj M, Hsu YF, Ajona D, Pio R, Blom AM. Non-small cell lung cancer cells produce a functional set of complement factor I and its soluble cofactors. Mol Immunol. 2008;45(1):169-179. doi:10.1016/j. molimm.2007.04.025

69. Riihila P, Nissinen L, Farshchian M, et al. Complement factor I promotes progression of cutaneous squamous cell carcinoma. J Invest Dermatol. 2015;135(2):579-588. doi:10.1038/jid.2014.376

70. Gasque P, Julen N, Ischenko AM, et al. Expression of complement components of the alternative pathway by glioma cell lines. J Immunol. 1992;149(4):1381-1387.

\section{Publish your work in this journal}

OncoTargets and Therapy is an international, peer-reviewed, open access journal focusing on the pathological basis of all cancers, potential targets for therapy and treatment protocols employed to improve the management of cancer patients. The journal also focuses on the impact of management programs and new therapeutic agents and protocols on

\section{Dovepress}

patient perspectives such as quality of life, adherence and satisfaction. The manuscript management system is completely online and includes a very quick and fair peer-review system, which is all easy to use. Visit http://www.dovepress.com/testimonials.php to read real quotes from published authors. 